\title{
REGSTATTOOLS: freeware statistical tools for the analysis of disease population databases used in health and social studies
}

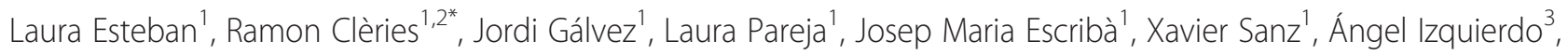
Jaume Galcerán ${ }^{4}$ and Josepa Ribes ${ }^{1,2}$

\begin{abstract}
Background: The repertoire of statistical methods dealing with the descriptive analysis of the burden of a disease has been expanded and implemented in statistical software packages during the last years. The purpose of this paper is to present a web-based tool, REGSTATTOOLS http://regstattools.net intended to provide analysis for the burden of cancer, or other group of disease registry data. Three software applications are included in REGSTATTOOLS: SART (analysis of disease's rates and its time trends), RiskDiff (analysis of percent changes in the rates due to demographic factors and risk of developing or dying from a disease) and WAERS (relative survival analysis).

Results: We show a real-data application through the assessment of the burden of tobacco-related cancer incidence in two Spanish regions in the period 1995-2004. Making use of SART we show that lung cancer is the most common cancer among those cancers, with rising trends in incidence among women. We compared 20002004 data with that of 1995-1999 to assess percent changes in the number of cases as well as relative survival using RiskDiff and WAERS, respectively. We show that the net change increase in lung cancer cases among women was mainly attributable to an increased risk of developing lung cancer, whereas in men it is attributable to the increase in population size. Among men, lung cancer relative survival was higher in 2000-2004 than in 1995-1999, whereas it was similar among women when these time periods were compared.

Conclusions: Unlike other similar applications, REGSTATTOOLS does not require local software installation and it is simple to use, fast and easy to interpret. It is a set of web-based statistical tools intended for automated calculation of population indicators that any professional in health or social sciences may require.
\end{abstract}

Keywords: Web-application, Prediction, Standardized incidence mortality ratio, Annual percent change, Net percent change of rates, Relative survival

\section{Background}

An aim of public health assessment involves describing the health status of a defined population by looking at their changes over time or by comparing their health events to events occurring in other populations. Descriptive epidemiology of cancer, for example, may assess the size of the problem that cancer poses to health, measuring

\footnotetext{
*Correspondence: r.cleries@iconcologia.net

${ }^{1}$ Cancer Registry of Catalonia, Plan for Oncology of the Catalan Government, IDIBELL, Hospital Duran i Reynals. Av. Gran Via de I'Hospitalet, 199-203, 08908 - L'Hospitalet de Llobregat, Catalonia, Spain

${ }^{2}$ Department of Clinical Sciences, University of Barcelona, Barcelona 08907, Spain

Full list of author information is available at the end of the article
}

the risk in the same population at different periods of time [1]. To account for rising trends of cancer in a population or to compare populations of different sizes, rates are usually developed to provide the number of events per population unit [2], whereas the number of cancer cases is used to measure the burden of cancer into the health system [3].

Worldwide, statistical methods for descriptive analysis has been expanded and implemented in statistical software packages during the last years. The most comprehensive coverage of statistical methods for analyzing cancer data is SEER"Stat [4], whereas user-friendly statistical software packages for specific time-trend modelling of rates have

\section{Biomed Central}

(c) 2013 Esteban et al.; licensee BioMed Central Ltd. This is an Open Access article distributed under the terms of the Creative Commons Attribution License (http://creativecommons.org/licenses/by/2.0), which permits unrestricted use, distribution, and reproduction in any medium, provided the original work is properly cited. 
also been developed to measure the burden of cancer and its projections [5-7] changes in trends [8], and survival analysis [9-11].

The purpose of this paper is to present a set of webbased tools, REGSTATTOOLS http://regstattools.net, in order to provide a very easy-intuitive way to carry out statistical analyses. The user must upload the predefined file to REGSTATTOOLS web-page to obtain for a determined disease and a period of time: (i) descriptive statistics, (ii) the estimated annual percent change in rates; (iii) the standardized incidence or mortality ratio comparing two time periods or two geographical areas; (iv) the prediction of the expected incident or death cases; (v) the assessment of the differences for incidence or deceased cases between two different time points or two geographical areas in order to clarify the role of the changes on demographic factors and the risk of developing or dying from the disease, and finally, (vi) comparing observed and relative survival.

In this paper REGSTATTOOLS is introduced describing its use through an example on the assessment of the burden of tobacco-related cancer incidence in two Spanish regions during the period 1995-2004.

\section{Implementation}

\section{Descriptive statistics for rates}

Suppose that we want to assess the burden of a disease in a certain population of size $\mathrm{N}$ during a certain period of time. Consider that we have observed $\mathrm{X}$ cases of the disease under study, therefore the crude rate $(\mathrm{CR})$ is defined as $\mathrm{X} / \mathrm{N}$. The $\mathrm{CR}$ is the simplest and most straightforward summary measure of the population's diseases under study. But the events may be strongly related to age, so the age-specific events will differ greatly from one another, therefore it is of interest to calculate the age-specific rates. The use of a world standard population [12] and direct standardization (or any other adjustment procedures) seek to provide numbers and comparisons that minimize the influence of age and/or other extraneous factors through the age-standardized rates (ASR) [13-15]. These ASRs can be also truncated for the age groups of interest. In cancer, the calculation of truncated rates (TR) over the age-range 35-64 [14] has been proposed, mainly because of doubts about the accuracy of age-specific rates in the elderly when diagnosis and recording of cancer may be much less certain. Finally, another useful summary measure of disease frequency is the cumulative rate $[14,15]$ (CumR), which is the sum of the age-specific incidence rates, taken from birth to age 74, in a certain time period. CumR is an estimate of the cumulative risk (Cumulative Risk $=1-\exp [-\mathrm{CumR}])$, which is the risk which an individual would have of developing an event of interest during a certain age-span if no other causes of death were in operation [14].
Estimating the annual percent change in rates (EAPC)

In descriptive epidemiology, the evolution of incidence or mortality rates of certain disease during a determined time period can generate etiological hypotheses. The estimated annual percent change (EAPC) is one way to characterize trends in disease rates over time. This means that the rates are assumed to change at a constant percentage of the rate of the previous year [15]. Let us assume that we want to assess the EAPC of ASRs during a period of time (in cancer, usually years). Let $A S R_{T}$ be the $A S R$ for the $\mathrm{T}^{\text {th }}$ year, $T$; the time trend of the ASRs can be modelled through a Gaussian log-linear model,

$$
\log \left(A S R_{T}\right)=\alpha+\beta \cdot T
$$

where $E A P C=\left(e^{\beta}-1\right) \cdot 100$. The $95 \%$ confidence intervals of the EAPC can be easily derived through the standard errors of model (1) [15].

\section{Predicting the Expected number of incident (or death)} disease cases by age group using the time trends of rates Prediction of future disease burden is essential for effective health service planning, as it may be utilized by public health authorities to formulate prevention, diagnosis and treatment strategies [16]. Simple log-linear models can be used to make these predictions [17]. If we assume that the time trend of $\frac{C_{i T}}{Y_{i T}}$, where $C_{i T}$ is the number of cases for the $i^{\text {th }}$ age-group and period $T$ and $Y_{i T}$ are the corresponding person-years at risk is linear in its log-scale, the following log-linear model can be fitted to these rates:

$$
\log \left(\frac{C_{i T}}{Y_{i T}}\right)=\alpha_{i}+\beta_{i} \cdot\left(T-T_{0}\right)
$$

where $T_{0}$ is the reference time, $\alpha_{i}$ is the log-rate at $T_{0}$ for the $i^{\text {th }}$ age-group and $\beta_{i}$ is the age-specific slope. A parsimonious version of this model can be also used assuming a common slope for each age group,

$$
\log \left(\frac{C_{i T}}{Y_{i T}}\right)=\alpha_{i}+\beta \cdot\left(T-T_{0}\right)
$$

where this model is known as the age-drift model. For these models we assume $C_{i T}$ to follow the Poisson distribution [17]. However, the negative binomial distribution has been also used as an alternative to Poisson when there is evidence of "overdispersion" (higher variance than expected) in the data [18].

Prediction of incidence at a future time $F$ can be made using the fitted model (2) or (3), and replacing $T$ by $F$ and $Y_{i T}$ by $Y_{i F}$ into the fitted model. Poisson and Negative Binomial distribution are both assumed for each model in (2) and (3). Therefore 4 models are assessed for the selection of the best fitting model to data. The assessment is 
made through the Akaike's Information Criterion (AIC) [19] and the Chi-square test [17].

\section{Comparing risk between two groups (time periods or geographical areas): standardized incidence or mortality ratio (SIMR)}

SIMR is used to determine if the occurrence of a disease in a target population is higher or lower than that occurrence in a reference one. For example, we can either compare the incidence of cancer in the same area in two different time periods or two different areas in the same time period. The SIMR can be calculated as

$$
\operatorname{SIMR}=\frac{D}{E}
$$

where $D$ is the number of observed events in the target population and $E$ the number of expected events in this population using the incidence (or mortality) rates of the reference population [15].

\section{Assessment of differences due to risk and demographic} factors when comparing disease rates of two populations To assess differences for incidence or deceased cases between two different time points or two areas in order to clarify the role of the changes in demographic factors and the risk of developing or dying from a disease, we used the method of Bashir and Estève [20]. For example, we can compare the observed age-specific incidence cases of certain cancers in the period 1995-1999, $C_{i}^{1995-1999}$, with the observed age-specific incidence cases in the period 2000-2004, $C_{i}^{2000-2004}$. Assuming eighteen 5-year agegroups, the observed percent net change of the difference in the total number of cases between both periods can be calculated as

$$
\operatorname{Net}(\%)=\frac{\sum_{i=1}^{18}\left(C_{i}^{2000-2004}-C_{i}^{1995-1999}\right)}{\sum_{i}^{18} C_{i}^{1995-1999}}
$$

Net(\%), can be separated into two components: i) changes in size and age distribution (structure) of the population and ii) changes in the risk of developing the disease,

$$
\begin{aligned}
\text { Net }(\%)= & \text { Risk }(\%)+\text { Population } \\
= & \text { Risk }(\%)+\text { Size }(\%) \\
& + \text { Structure }(\%)
\end{aligned}
$$

We note that in each age group we must take into account that rates into the period 2000-2004 must be considered as constant as well as rates into the period 1995-1999. If the population size is expected to increase by $10 \%$, incident cases will also increase by
$10 \%$. The effect of population structure is estimated by comparing the rate observed in 1995-1999 and the rate expected in the 2000-2004, through applying the age specific rates observed in 1995-1999 to the population pyramid in 2000-2004. Lastly, the percent change not explained by percent change in the population will be considered to be due to the variation in risk of developing the disease [20]. We note that the net change can be also calculated for the CR [20]. Mathematical details of equation (6) can be found in the Additional file 1.

\section{Assessing survival of a cohort of patients diagnosed with a certain disease}

The observed survival (OS) rate is the basic measure of the survival experience of a group of patients from the date of diagnosis to a certain time. However, information on the cancer patients' causes of death might not be always suitable or it might be vague or unavailable [15,21]. Since the interest lies in describing mortality attributable to the disease under study, one method of estimating net survival, where the disease of interest is assumed to be the only possible cause of death [21], is relative survival (RS). It is interpreted as the probability to survive after diagnosis of the disease of interest. For a cohort of patients diagnosed with a certain disease, say cancer for example, the cumulative RS at time $T$ is defined as

$$
R S(T)=\frac{S_{O}(T)}{S_{E}(T)}
$$

where $S_{o}(T)$ is the observed survival rate in the cohort of study and $S_{E}(T)$ is the expected survival of that cohort, this last estimated from a comparable general population life tables stratified by age, sex and calendar time and assume that the cancer deaths are a negligible proportion of all deaths [21]. The $R S(T)$ can be calculated through estimating $S_{o}(T)$ by the Kaplan-Meier method and $S_{E}(T)$ using Hakulinen method [22]. The 95\% confidence intervals of the $R S(T)$ can be estimated through the standard errors of the log-transformation of $S_{o}(T)$ assuming $S_{E}(T)$ as a constant value [23]. Some interpretations about RS are not straightforward. Note that improvements in general mortality of the reference population affect $S_{E}(T)$ in Equation (7) [24]. Let's suppose we want to compare 5-year RS of lung cancer between periods $1999-1994 \quad(\operatorname{RS}(5)=10,5 \%)$ and 1995-1999 (RS(5)=8,5\%) among men in Catalonia (Spain) [25]. Although cancer mortality decreased in 2000-2004 compared to 1995-1999 in Catalonia [26], we observed a decrease of 5-year RS of lung cancer. It may suggest that RS(5) was worsening in 1995-1999 but the explanation is that $S_{E}(5)$ between both periods increased but $S_{o}(5)$ remained stable, and therefore RS(5) decreased [26]. In this line, two period comparison of RS for cancers with poor survival should be interpreted with caution [24]. 
The set of web-based applications included in REGSTATTOOLS

REGSTATTOOLS (http://regstattools.net/) includes a set of web-based statistical applications running under Linux operating system installed in a web-server: SART (Statistical Analysis of Rates and Trends) [27], RiskDiff (a web tool for the analysis of the difference due to risk and demographic factors for incidence or mortality data) [28] and WAERS (Web-Assisted Estimation of Relative Survival) [29]. The web pages of all these applications were implemented using the server-side scripting language $P H P$ and HTML [30] whereas statistical computation has been implemented using $R$ statistical software [31].

Figure 1 shows an overview of the REGSTATTOOLS applications. Each application requires at least one input file to perform the corresponding statistical analysis. The user must have an Individual Records data file available which is a basis file to perform all the statistical analysis, since most of the data files used in the web applications are derived from this one. This basis file must contain the following patients' variables: patient identification, sex, type of disease, age-month-year at diagnosis, status of the patient (dead or alive), month-year of the death, and finally, the follow-up time in years. The SART applications require a total of 6 files to be uploaded whereas RiskDiff and WAERS applications only require one file each.

The SART applications [27] require an Aggregated Data file that must contain the following columns: sex, age-group, incidence or mortality year, type of disease, cases and person-years at risk. To perform a descriptive analysis of the disease rates, the user can make use of the Descriptive application after preparing an age-groups file and a standard population's file. The time trends analysis of rates can be performed using the EAPC application which also requires a standard population file. The application Expected allows a prediction of the expected number of cases in a future period or in other

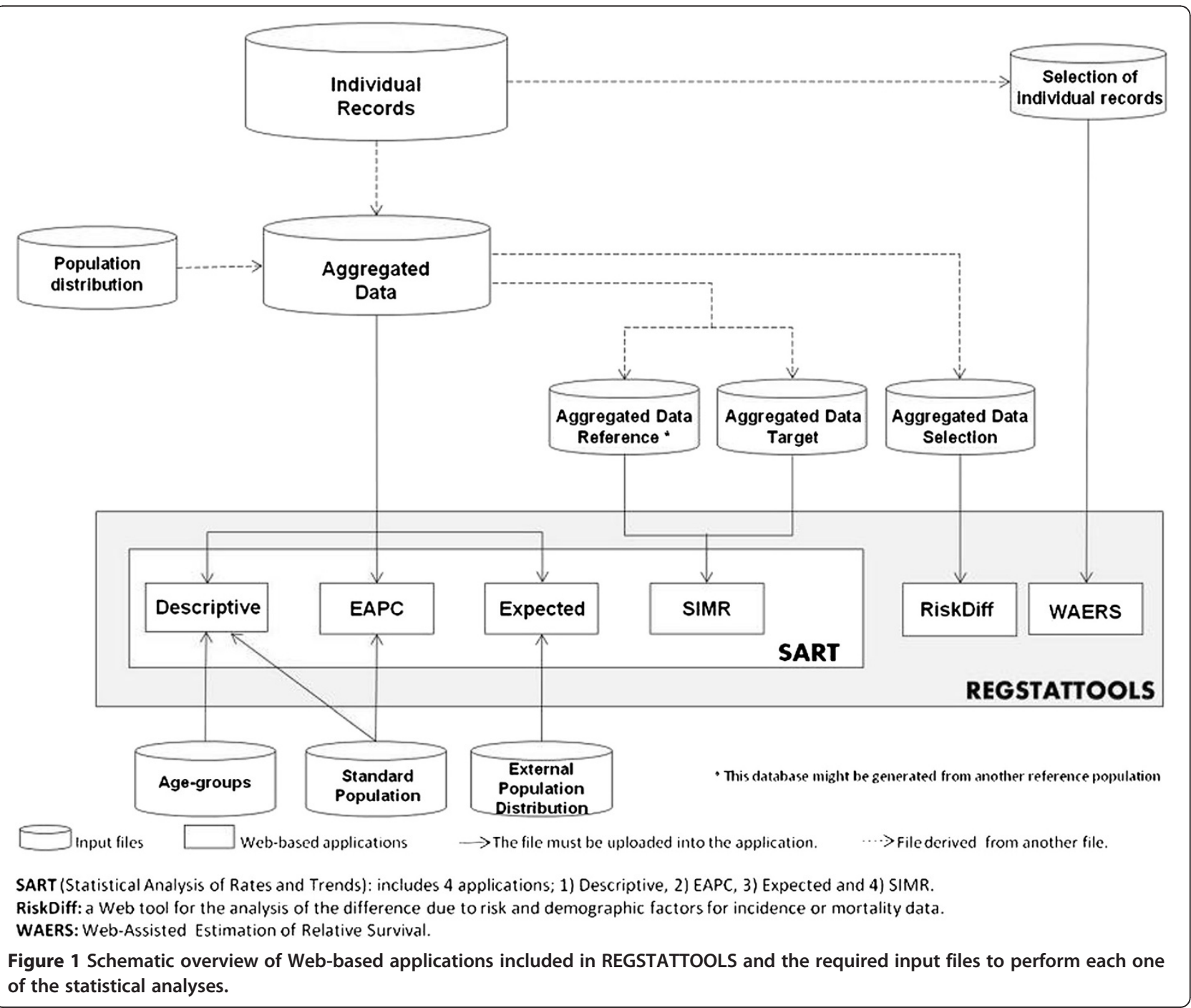


geographical areas, using the aggregated data and a file which contains an external population distribution (person-years at risk by age-group). The comparison of risk between two groups can be performed through SIMR application which requires a partition of the $A g$ gregated Data file into two files, each one with data of the corresponding time period. Another possibility could be comparing these data with data from another area in the same time period; therefore, two files are required. In this line, note that the user must prepare 6 files to fully run $S A R T$.

The RiskDiff application [28] has been developed to perform the analysis described in section Assessment of differences due to risk and demographic factors when comparing disease rates of two populations. It requires information on the number of cases and person years at risk by age-group in each one of the two periods or two geographical areas to be compared. In this line, the $A g$ gregated Data Selection file must contain 2 columns for each period/area compared: one column referring to person-years and another column referring to number of cases.

Finally, the RS must be obtained through the WAERS application [29] which requires a Selection of Individual Records file with the following variables: patients ID, age and year at beginning of study, sex, years of follow-up and vital status (death or alive).

We will refer to AF throughout the paper where additional figures and tables can be found, and those that are related to the example section.

\section{Results}

To illustrate the use of REGSTATTOOLS we will assess the burden of tobacco-related cancer in Girona and Tarragona provinces (northeast of Spain), which cover 1,200,000 inhabitants in 2001. Incidence data were provided in the Individual Records data file which includes data from all patients diagnosed with larynx, oral cavity, pharynx, oesophagus, stomach, lung, pancreas and kidney tumours in the period 1995-2004. Patients were followed-up until December 31st 2007. A total 10297 men and 2695 women (mean age 66.1 and 70.1 years, respectively) were included in the analysis. This file is the basis to perform the statistical analyses (Table 1) from which we can create the data file to be uploaded into SART by means of aggregated data in eighteen 5-year age-groups (or other age groups according to user's requirements).

The Descriptive application has been used after preparing an age-groups file (Additional file 1: Table Aff1) and a world standard population's file (Additional file 1: Table Aff2). Since we are analysing rates, note that Girona and Tarragona population's person-years at risk for each sexage-group-year are required. We made use of the Catalan Institute of Statistics population's distribution (available at: www.idescat.net). Merging a Population-Distribution (person-years) data file (Additional file 1: Table Aff3) and the previous one, we can obtain the Aggregated Data file (Table 2). Results of the descriptive analysis of tobacco-related cancer in Girona and Tarragona showed that ASR of lung cancer is the first ranking in men's $(\mathrm{ASR}=48.27$ per 100,000 men-years at risk) and the second

Table 1 File example of individual records

\begin{tabular}{|c|c|c|c|c|c|c|c|c|c|}
\hline Patient_ID & Sex & d_group & d_age & i_month & i_year & f_month & f_year & Status & Follow_up \\
\hline 1 & 1 & Lung & 84 & 3 & 1995 & 3 & 1995 & 1 & 0.08 \\
\hline 2 & 1 & Lung & 65 & 2 & 1995 & 12 & 1999 & 1 & 0.83 \\
\hline 3 & 1 & Lung & 63 & 3 & 1995 & 5 & 1999 & 1 & 4.17 \\
\hline$\ldots$ & $\ldots$ & $\ldots$ & $\ldots$ & $\ldots$ & $\ldots$ & $\ldots$ & $\ldots$ & $\ldots$ & $\ldots$ \\
\hline 194 & 2 & Lung & 72 & 2 & 1995 & 8 & 1995 & 1 & 0.50 \\
\hline 195 & 2 & Lung & 42 & 9 & 1995 & 11 & 1995 & 1 & 0.17 \\
\hline 196 & 1 & Lung & 72 & 10 & 1995 & 1 & 1996 & 1 & 0.25 \\
\hline 197 & 1 & Lung & 52 & 5 & 1995 & 12 & 2008 & 0 & 12.58 \\
\hline 198 & 1 & Lung & 79 & 1 & 1996 & 9 & 1997 & 1 & 1.67 \\
\hline$\ldots$ & $\ldots$ & $\ldots$ & $\cdots$ & $\ldots$ & $\cdots$ & $\cdots$ & $\ldots$ & $\cdots$ & $\cdots$ \\
\hline 6087 & 1 & Larynx & 59 & 6 & 2000 & 8 & 2004 & 1 & 4.17 \\
\hline 6088 & 2 & Larynx & 68 & 7 & 2001 & 8 & 2004 & 1 & 3.08 \\
\hline 6089 & 2 & Larynx & 53 & 3 & 2000 & 12 & 2006 & 0 & 6.75 \\
\hline 6090 & 1 & Larynx & 87 & 7 & 1995 & 12 & 2006 & 0 & 11.42 \\
\hline$\ldots$ & $\ldots$ & $\ldots$ & $\ldots$ & $\ldots$ & $\ldots$ & $\ldots$ & $\ldots$ & $\ldots$ & $\ldots$ \\
\hline 12989 & 1 & Kidney & 50 & 7 & 2002 & 5 & 2003 & 1 & 0.83 \\
\hline 12990 & 2 & Kidney & 90 & 7 & 2003 & 7 & 2003 & 1 & 0.08 \\
\hline 12991 & 1 & Kidney & 49 & 6 & 2004 & 6 & 2004 & 1 & 0.08 \\
\hline 12992 & 1 & Kidney & 67 & 4 & 2004 & 6 & 2004 & 1 & 0.08 \\
\hline
\end{tabular}


Table 2 Girona and Tarragona aggregated data

\begin{tabular}{rrrrrr}
\hline Sex & Age.group & Year & Group & Cases & Population \\
\hline 1 & 1 & 2000 & Kidney & 0 & 27251 \\
\hline 1 & 2 & 2000 & Kidney & 0 & 27741 \\
\hline 1 & 3 & 2000 & Kidney & 0 & 29381 \\
\hline$\cdots$ & $\cdots$ & $\ldots$ & $\cdots$ & $\cdots$ & $\cdots$ \\
\hline 2 & 16 & 2004 & Larynx & 0 & 26115 \\
\hline 2 & 17 & 2004 & Larynx & 0 & 19665 \\
\hline 2 & 18 & 2004 & Larynx & 0 & 15737 \\
\hline$\cdots$ & $\cdots$ & $\ldots$ & $\cdots$ & $\cdots$ & $\cdots$ \\
\hline 1 & 1 & 2000 & Lung & 0 & 27251 \\
\hline 1 & 2 & 2000 & Lung & 0 & 29381 \\
\hline$\cdots$ & $\cdots$ & $\cdots$ & $\cdots$ & $\cdots$ & $\cdots$ \\
\hline 2 & 16 & 2004 & Stomach & 8 & 26115 \\
\hline 2 & 17 & 2004 & Stomach & 19 & 19665 \\
\hline 2 & 18 & 2004 & Stomach & 15 & 15737 \\
\hline
\end{tabular}

one in women's (ASR=5.12 per 100,000 women-years at risk) in the ranking of the tumours of interest (Additional file 1: Figure Aff1). Lung cancer has the highest risk among the tobacco-related cancers in men (CumR $=6 \%$, 6 per 100 men are at risk of developing lung cancer before 75 years old, Additional file 1: Table Aff4). In women, lung and stomach cancers have the highest risk among the tobacco-related cancers $(\mathrm{CumR}=0.58 \%$, less than 1 per
100 women are at risk of developing lung or stomach cancer before 75 years old, Additional file 1: Table Aff4).

EAPC of tobacco-related cancer rates during the period 1995-2004 in both sexes is depicted in Figure 2. EAPC can be interpreted as the annual change of rates in the magnitude of a trend. If the confidence interval includes 0 value, the change is not statistically significant whereas if it is not included, it is interpreted as a significant increase (if it is positive) or decrease (if it is negative). In men, lung cancer remained stable $(\mathrm{EAPC}=-0.24,95 \% \mathrm{CI}:-1.54,1.08)$ whereas some tumours showed a significant decreasing trend such as stomach $(\mathrm{EAPC}=-4.14,95 \% \mathrm{CI}:-6.79,-1.42)$ and oropharynx $(\mathrm{EAPC}=-3.23,95 \% \mathrm{CI}:-5.43,-0.97)$ and $\mathrm{a}$ significant increasing trend such as pancreas $(\mathrm{EAPC}=2.77$, 95\%CI: 0.73, 4.85) (Additional file 1: Table Aff5). In women, significant increases in the study period were detected in lung (EAPC $=6.16,95 \% \mathrm{CI}: 1.81,10.70)$ and oropharynx tumours (EAPC $=3.52$, 95\%CI: 0.91, 6.19), (Additional file 1: Table Aff5).

Although some cancers did not show a significant time trend during the whole time period, there might be a change in the risk of developing the cancer at the individual level. Therefore, we could prepare two datasets, one which includes 1995-1999 aggregated data (reference period) and another with 2000-2004 aggregated data (target period) (Additional file 1: Table Aff6) and making use of the SIMR application we could assess the change in risk of developing lung cancer between

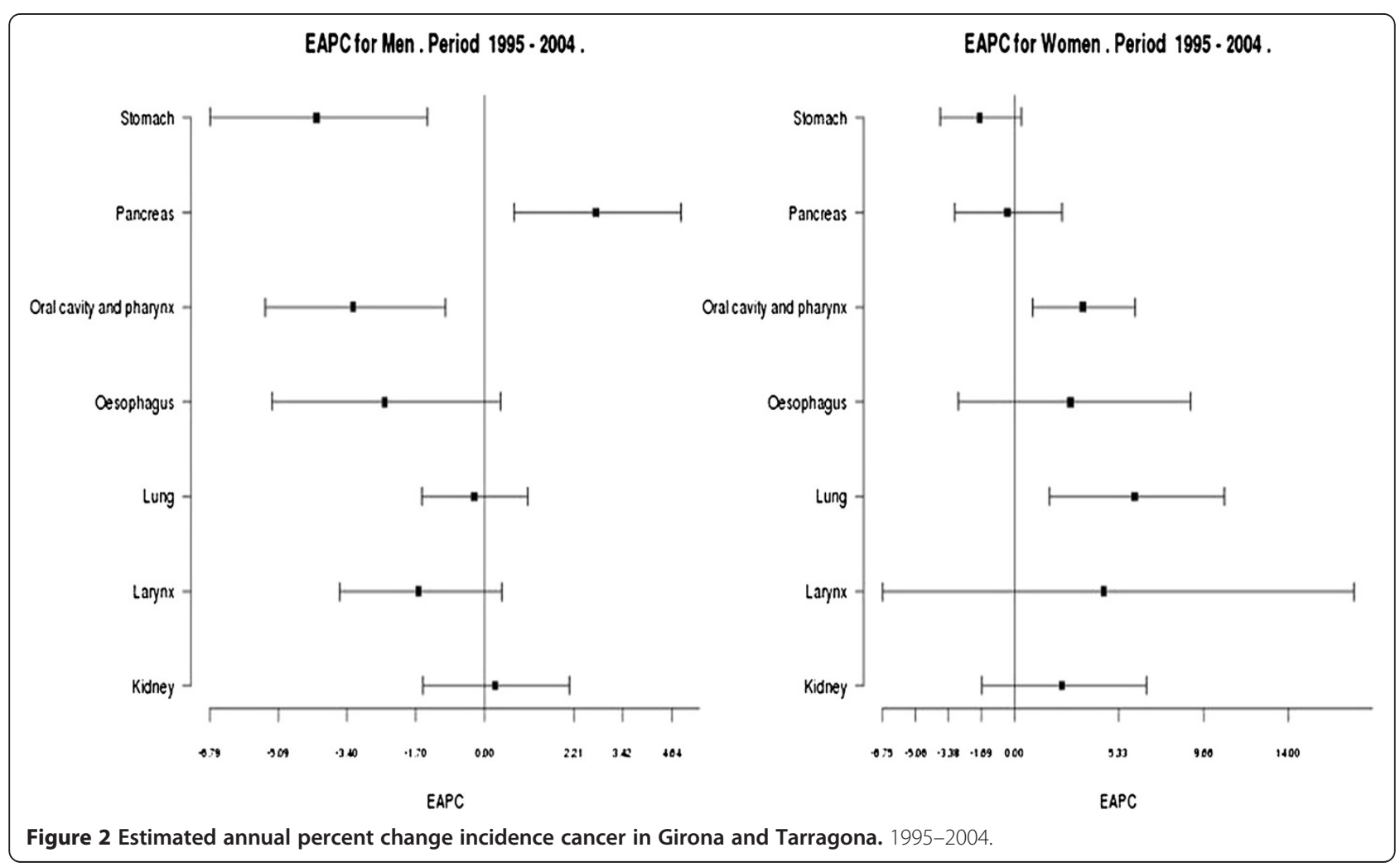


these periods. SIMR can be interpreted as the increase or decrease of risk of developing or of dying from a disease comparing the observed number of cases of a population respect to a reference one. If the SIMR's confidence interval includes the value 1 , the SIMR is not statistically significant, otherwise, it is interpreted as a significant excess or reduced risk of having a disease or dying from a disease. Figure 3 compares the SIMRs for each cancer site analyzed between men and women. In women, a significant $17 \%$ higher lung cancer incidence is observed with 2000-2004 versus 1995-1999 (SIMR=1.17, 95\% CI: 1.04, 1.30, Additional file 1: Table Aff7).

To assess the changes in the number of incident lung cancer cases among time periods making use of RiskDiff we have prepared an Aggregated Data Selection file (Additional file 1: Table Aff8). Assuming that incidence rates in the period 2000-2004 are constant, as well as rates in the period 1995-1999 are also constant, we have compared cancer incidence in both periods. We found that the number of cases was $11.63 \%$ higher in 2000 2004 compared to 1995-1999 among men, whereas among women this change was about $30.0 \%$ (Additional file 1: Table Aff9). Figure 4 shows the contribution of demographic factors and the risk of developing lung cancer to this net change in the CR, cases per 100,000 person-years, and absolute number of cases. In men, changes in the CR due to risk and population structure are similar (Risk: 0.75 and Structure: 0.78 cases per
100,000 person-years) whereas changes in risk contribute the most among women (Risk: 1.52 and Structure: 0.24 cases per 100,000 person-years). In men net change in absolute number of cases was basically due to a change in population size $(9.78 \%)$, while changes in population structure and changes in the risk of developing lung cancer were much less important, $0.95 \%$ and $0.91 \%$ respectively. On the contrary, in women, the net change in the absolute number of cases was mainly attributable to an increased risk of developing lung cancer (17.53\%) while the changes in size and population structure play a less important role ( $9.76 \%$ and a $2.72 \%$, respectively) (Additional file 1: Table Aff9 and Figure 4). These results are in agreement with those observed in the SIMR analyses.

We also assessed the evolution of the 5-year RS rates of lung cancer between the time period 2000-2004 and the time period 1995-1999 using WAERS through a Selection of Individual Records file (Additional file 1: Table Aff10). Table 3 shows the WAERS output, where we found that 5-year RS improved significantly among men (5-year RS $1995-1999=8.3 \%$, 95\% CI: $7.1 \%-9.6 \%$ versus 5 -year RS 2000-2004=11.5\%, 95\% CI: 10.1\%-13.0\%) whereas these differences between RS were not statistically significant among women (5-year RS 1995-1999=10.6\%, 95\% CI: $7.1 \%-15.7 \%$ versus 5 -year RS $2000-2004=14.0 \%$, 95\% CI: $10.4 \%-18.9 \%)$.

Finally, we predicted the burden of lung cancer for the year 2014 in Catalonia through the Expected application

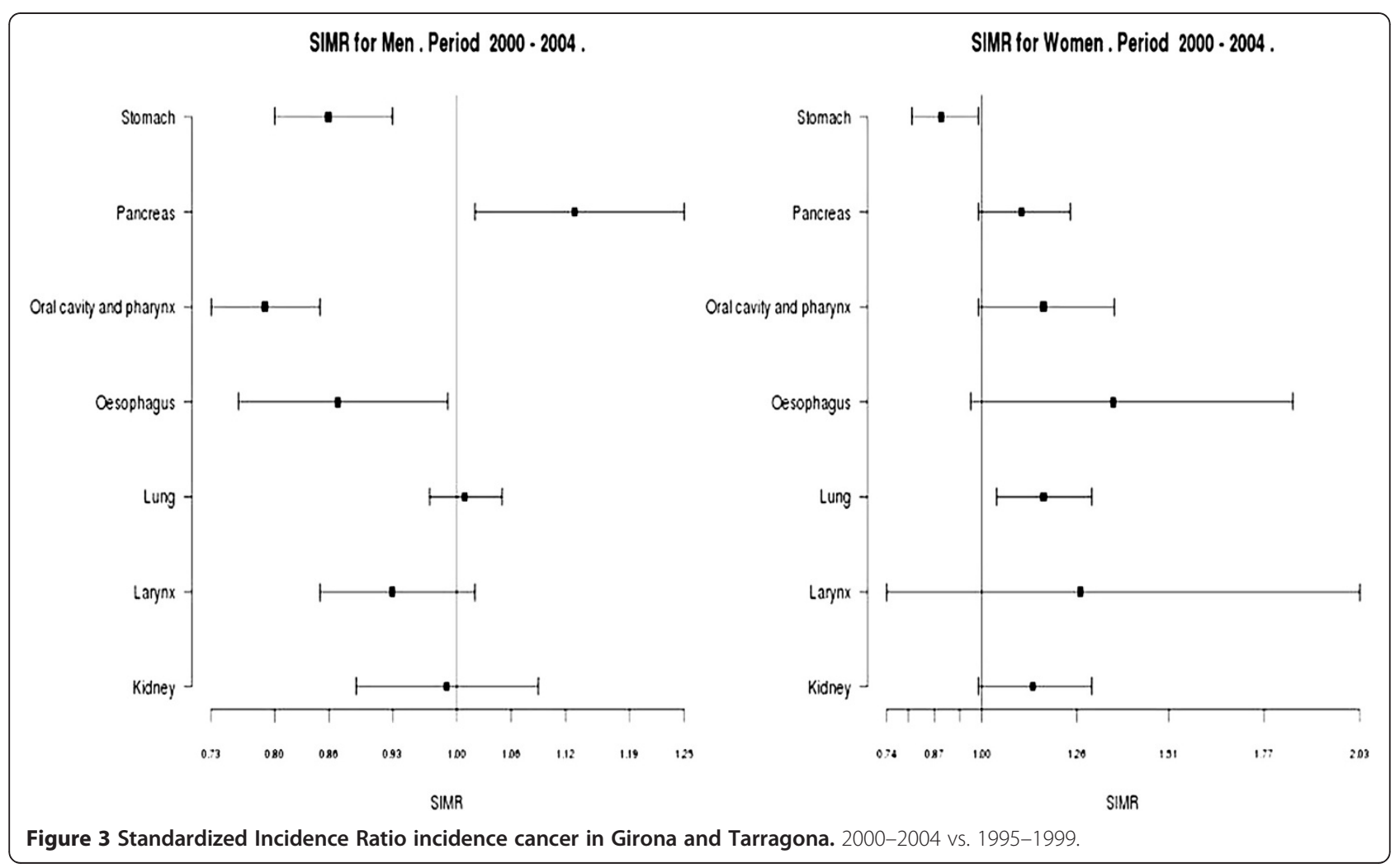


Table 3 WAERS output for lung cancer incidence in Girona and Tarragona. 4 executions by period and sex

\begin{tabular}{|c|c|c|c|c|c|c|c|c|c|c|c|}
\hline \multicolumn{12}{|c|}{ Men } \\
\hline \multicolumn{6}{|c|}{ 1995-1999 } & \multicolumn{6}{|c|}{$2000-2004$} \\
\hline Risk & $T$ & RS & $\mathrm{LCl}$ & $\mathrm{UCl}$ & OS & Risk & $\mathrm{T}$ & RS & $\mathrm{LCl}$ & $\mathrm{UCl}$ & $\overline{O S}$ \\
\hline 2252 & 0 & 0.999 & 0.998 & 1 & 0.999 & 2514 & 1 & 0.325 & 0.307 & 0.344 & 0.313 \\
\hline 2250 & 1 & 0.292 & 0.273 & 0.312 & 0.282 & 775 & 2 & 0.191 & 0.175 & 0.207 & 0.179 \\
\hline 616 & 2 & 0.156 & 0.141 & 0.173 & 0.146 & 436 & 3 & 0.15 & 0.135 & 0.165 & 0.136 \\
\hline 319 & 3 & 0.113 & 0.1 & 0.128 & 0.103 & 299 & 4 & 0.129 & 0.116 & 0.144 & 0.114 \\
\hline 224 & 4 & 0.096 & 0.084 & 0.11 & 0.085 & 219 & 5 & 0.115 & 0.101 & 0.13 & 0.099 \\
\hline 186 & 5 & 0.083 & 0.071 & 0.096 & 0.071 & 140 & 6 & 0.106 & 0.093 & 0.121 & 0.089 \\
\hline 155 & 6 & 0.074 & 0.063 & 0.087 & 0.062 & 89 & 7 & 0.101 & 0.087 & 0.117 & 0.082 \\
\hline 135 & 7 & 0.066 & 0.055 & 0.079 & 0.054 & 40 & 8 & 0.089 & 0.073 & 0.108 & 0.07 \\
\hline 117 & 8 & 0.056 & 0.046 & 0.068 & 0.044 & 17 & 9 & 0.065 & 0.045 & 0.093 & 0.049 \\
\hline 89 & 9 & 0.051 & 0.042 & 0.063 & 0.039 & & & & & & \\
\hline 61 & 10 & 0.047 & 0.037 & 0.059 & 0.035 & & & & & & \\
\hline 41 & 11 & 0.04 & 0.031 & 0.052 & 0.029 & & & & & & \\
\hline 21 & 12 & 0.037 & 0.028 & 0.05 & 0.026 & & & & & & \\
\hline 12 & 13 & 0.036 & 0.025 & 0.05 & 0.024 & & & & & & \\
\hline 4 & 14 & 0.027 & 0.014 & 0.053 & 0.018 & & & & & & \\
\hline \multicolumn{12}{|c|}{ Women } \\
\hline \multicolumn{6}{|c|}{ 1995-1999 } & \multicolumn{6}{|c|}{$2000-2004$} \\
\hline Risk & $T$ & RS & $\mathrm{LCl}$ & $\mathrm{UCl}$ & OS & Risk & $\mathrm{T}$ & RS & $\mathrm{LCl}$ & $\mathrm{UCl}$ & OS \\
\hline 240 & 1 & 0.275 & 0.222 & 0.339 & 0.267 & 312 & 1 & 0.339 & 0.289 & 0.397 & 0.33 \\
\hline 61 & 2 & 0.174 & 0.131 & 0.232 & 0.166 & 100 & 2 & 0.227 & 0.184 & 0.281 & 0.218 \\
\hline 38 & 3 & 0.135 & 0.097 & 0.189 & 0.127 & 65 & 3 & 0.174 & 0.135 & 0.224 & 0.164 \\
\hline 29 & 4 & 0.123 & 0.086 & 0.177 & 0.114 & 42 & 4 & 0.147 & 0.11 & 0.196 & 0.137 \\
\hline 26 & 5 & 0.103 & 0.071 & 0.157 & 0.096 & 31 & 5 & 0.14 & 0.104 & 0.189 & 0.128 \\
\hline 22 & 6 & 0.093 & 0.061 & 0.142 & 0.083 & 20 & 6 & 0.128 & 0.092 & 0.179 & 0.115 \\
\hline 19 & 8 & 0.085 & 0.054 & 0.033 & 0.074 & 9 & 7 & 0.073 & 0.037 & 0.143 & 0.064 \\
\hline 15 & 9 & 0.08 & 0.05 & 0.128 & 0.069 & 1 & 9 & 0.086 & 0.044 & 0.169 & 0.064 \\
\hline 12 & 10 & 0.08 & 0.05 & 0.129 & 0.069 & & & & & & \\
\hline 7 & 11 & 0.082 & 0.051 & 0.031 & 0.069 & & & & & & \\
\hline 3 & 12 & 0.083 & 0.052 & 0.134 & 0.069 & & & & & & \\
\hline 1 & 13 & 0.088 & 0.055 & 0.141 & 0.069 & & & & & & \\
\hline
\end{tabular}

of SART making use of the predicted population for Catalonia in 2014 (Additional file 1: Table Aff11). The application selected the age-drift models (Additional file 1: Table Aff12) as the best fitting ones, predicting 777 cases among women with an ASR of 10.74 cases per 100,000 women-years and 3085 cases among men with an ASR of 46.82 cases per 100,000 men-years (Additional file 1: Table Aff13). We can also observe the cases by age-group in Additional file 1: Table Aff14 (the output of the application).

\section{Discussion}

There are many "stand-alone" web pages which are designed to perform only a single statistical test or calculation. REGSTATTOOLS is a website which performs an entire suite of calculations for registry data, with a logical organization and consistent user interface. REGSTATTOOLS incorporates a flexible data import with a variety of methods in order to facilitate the widespread use of these applications with a basic statistical knowledge. The development of REGSTATTOOLS applications is an ongoing process which has been implemented with the current version of SART, RiskDiff and WAERS. In this paper, the use of REGSTATTOOLS' applications was illustrated by analyzing populationbased cancer registry data. However, it can be used to analyze other disease registry data such as diabetes or any other chronic disease.

Up to date of publication SART, WAERS and RiskDiff were accessed 3,179 times in total. Although each 


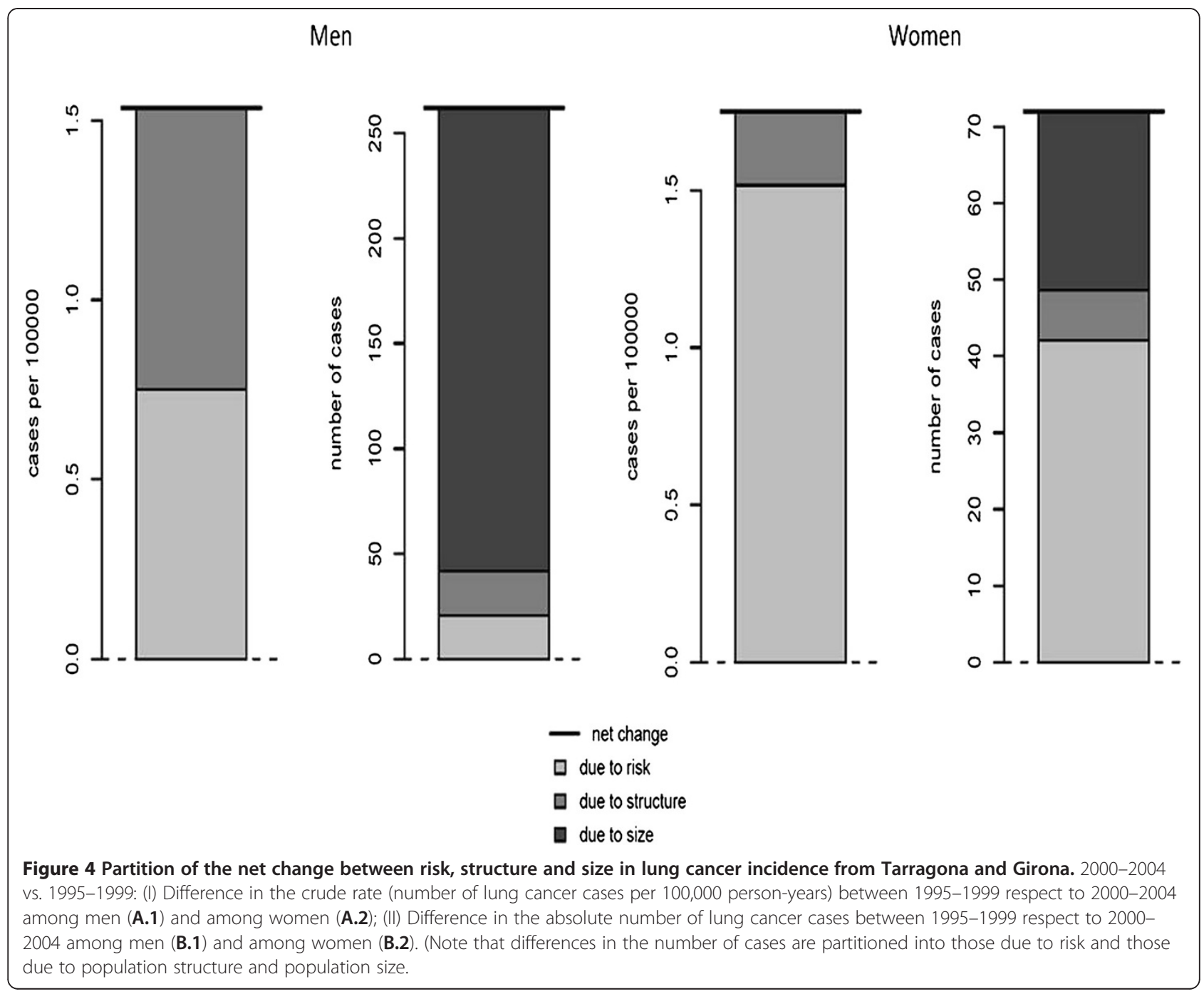

application was available on the internet at a different period of time, each one shows different percentage of use by user type (see Additional file 1: Table Aff15). Cancer registries are the main users of $S A R T(65.94 \%)$ and WAERS (60.71\%) whereas they are the second in the ranking of RiskDiff (35.71\%) users. Universities and research centres are also major WAERS (26.16\%) and RiskDiff $(42.86 \%)$ users.

$S A R T$ includes the calculation of disease rates and other indicators in the same application with no requirement of software installation [27]. WAERS is a web-based survivalspecific application to perform basic RS analysis [29]. Nowadays, mortality rates from all European countries, the United States of America, Canada and Argentina have been incorporated into WAERS. Any WAERS user from all these countries can estimate RS making use of these mortality tables. More advanced survival analysis can be carried out using other statistical software [9-11] that requires previous installation in the user's local computer as well as some technical skills about multivariate analysis. On the other hand and to our knowledge, RiskDiff is the only available web tool that can perform a statistical analysis which identifies which percentage of change in disease rates between two time periods or areas are due to changes in demographic factors, and which are due to changes in risk [28].

Some limitations should be noted in these applications in their current versions. SART does not provide confidence intervals for rates, and axis limits of graphs are created automatically by the application. WAERS does not allow a comparison of two or more RS curves since it does not compute RS for two or more groups at the same time. RiskDiff does not allow the assessment of statistical significance for the percent changes in risk and demographics. Future work will incorporate the integration of these features within these applications, although research in statistical methods must be developed specifically for RiskDiff. 


\section{Conclusions}

Unlike other similar applications, REGSTATTOOLS does not require local software installation and it is simple to use, fast and easy to interpret. REGSTATTOOLS is a set of web-based statistical tools intended for automated calculation of population indicators that any professional in the health or social sciences may require.

\section{Availability and requirements \\ Project name: REGSTATTOOLS. \\ Project home page: Access to the set of applications SART, RiskDiff and WAERS can be found through http:// regstattools.net/.}

Operating system: Platform independent for accessing the public web server.

Programming language: $\mathrm{R}$ and PHP.

Requirements: $\mathrm{R}$ statistical software available at http:// www.r-project.org/ website is required for the functions implemented.

License: None.

Any restriction to use by non-academics: None.

\section{Additional file}

Additional file 1: "REGSTATTOOLS: freeware statistical tools for the analysis of disease population databases used in health and social studies".

\section{Abbreviations \\ ASR: Age standardized rate; CR: Crude rate; TR: Truncated rate; cumR: Cumulative rate; EAPC: Estimated annual percent of change; OS: Observed survival; RS: Relative survival; SIMR: Standardized incidence or mortality ratio; AF: Additional file; 95\%Cl: 95\% confidence interval; RiskDif: A web tool for the analysis of the difference due to risk and demographic factors for incidence or mortality data; SART: Statistical analysis of rates and trends; WAERS: Web-assisted estimation of relative survival.}

\section{Competing interests}

The authors declare that they have no competing interests.

\section{Authors' contributions}

$L E, R C, J G, L P$ and JR initially conceived the tool and were involved in its design. $L E, R C$ and $L P$ did the statistical analysis and the implementation in $R$ JG implemented the web interface. JG and Al contributed to the collection, processing and interpretation of the data. All authors have been involved in drafting the manuscript and revising it critically. All authors approved the final version.

\section{Acknowledgements}

We would like to thank the two reviewers as well as the editor for their careful reviews and constructive comments that have improved the manuscript significantly. We would like to thank Dr. Genevieve Buckland for the very constructive and helpful comments. This work was supported by a UICC International Cancer Technology Transfer Fellowship and with Federal funds from the National Cancer Institute, National Institutes of Health under Contract NO2-CO-41101.

\section{Author details}

${ }^{1}$ Cancer Registry of Catalonia, Plan for Oncology of the Catalan Government, IDIBELL, Hospital Duran i Reynals. Av. Gran Via de l'Hospitalet, 199-203, 08908 - L'Hospitalet de Llobregat, Catalonia, Spain. ${ }^{2}$ Department of Clinical Sciences, University of Barcelona, Barcelona 08907, Spain. ${ }^{3}$ Epidemiology Unit and Cancer Registry of Girona, Institut Català d'Oncologia (ICO), Girona
17004, Catalonia, Spain. ${ }^{4}$ Tarragona Cancer Registry. Foundation Society for Cancer research and Prevention, Pere Virgili Health Research Institute, Reus 43201, Spain.

Received: 24 July 2012 Accepted: 27 February 2013

Published: 7 March 2013

\section{References}

1. Tomatis L, Aitio A, Day N, Heseltine E, Kaldor J, Miller A, Parkin D, Riboli E: Cancer: causes,occurrence and control. Lyon: IARC Scientific Publications Nr 100; 1990.

2. Esteve J, Benhamou E, Raymond L: Statistical methods in cancer research. Descriptive epidemiology. IARC Sci Publ 1994, IV:1-302.

3. Bray F, Moller B: Predicting the future burden of cancer. Nat Rev Cancer 2006, 6:63-74,

4. Surveillance Research Program, N. C. I. S. s. v. 7. 0. 5: Surveillance research program, national cancer institute SEER*stat software. www.seer.cancer.gov/ seerstat. 2011. 17-12-0012 Ref Type: Electronic Citation.

5. De Angelis G, De Angelis R, Frova L, Verdecchia A: MIAMOD: a computer package to estimate chronic disease morbidity using mortality and survival data. Comput Methods Programs Biomed 1994, 44:99-107.

6. Verdecchia A, De Angelis G, Capocaccia R: Estimation and projections of cancer prevalence from cancer registry data. Stat Med 2002, 21:3511-3526.

7. Schmid V, Held L: Bayesian age-period-cohort modeling and predictionBAMP. J Stat Softw 2007, 21(8):1-15.

8. Kim HJ, Fay MP, Feuer EJ, Midthume DN: Permutation tests for joinpoint regression with applications to cancer rates. Stat Med 2000, 19:335-351.

9. Dickman PW, Hakulinen T, Voutilaninen ET: SURV3, Relative survival analysis. 2000. Ref Type: Unpublished Work.

10. Yu B, Tiwari RC, Cronin KA, McDonald C, Feuer EJ: CANSURV: a windows program for population-based cancer survival analysis. Comput Methods Programs Biomed 2005, 80:195-203.

11. Geiss K, Meyer M, Radespiel-Troger M, Gefeller O: SURVSOFT-Software for nonparametric survival analysis. Comput Methods Programs Biomed 2009, 96:63-71.

12. Segi M, FUKUSHIMA I, Kurihara M: A proposal on a calculation method to be applied by geographical comparison of cancer mortality. Tohoku J Exp Med 1954, 60:307-310.

13. Rothman KJ, Greenland S: Modern epidemiology. Philadelphia: Lippincott Williams \& Wilkins; 1998.

14. Bray F: Age-standardization. In Cancer incidence in five continents, volume VIII. IARC scientific publications No. 155. Edited by Parkin DM, Whelan SL, Ferlay J, Teppo L, Thomas DB. Lyon: International Agency for Research on Cancer; 2002:87-89.

15. Estève J, Benhamou E, Raymond L: Statistical Methods in Cancer Research (Vol. IV). Lyon: IARC Scientific Publications, No 128; 1994.

16. Hakulinen T, Hakama M: Predictions of epidemiology and the evaluation of cancer control measures and the setting of policy priorities. Soc Sci Med 1991, 33:1379-1383.

17. Dyba T, Hakulinen T: Comparison of different approaches to incidence prediction based on simple interpolation techniques. Stat Med 2000, 19:1741-1752.

18. McCullagh P, Nelder J: Generalized linear models. London: Chapman \& Hall; 1989.

19. Akaike H: IEEE transactions on automatic control 19. IEEE Trans Autom Control 1974, 19(6):716-723.

20. Bashir SA, Estève J: Projecting cancer incidence and mortality using Bayesian age-period-cohort models. J Epidemiol Bioestat 2001, 6:287-296.

21. Ederer $F$, Axtell LM, Cutler SJ: The relative survival rate: a statistical methodology. Natl Cancer Inst Monogr 1961, 6:101-121.

22. Hakulinen T: Cancer survival corrected for heterogeneity in patient withdrawal. Biometrics 1982, 38:933-942.

23. Cleries R, Ribes J, Moreno V, Esteban L, Pareja L, Galvez J, Martinez JM, Bosch FX, Borras JM: Relative survival computation. Comparison of methods for estimating expected survival. Gac Sanit 2006, 20:325-331.

24. Perme MP, Stare J, Esteve J: On estimation in relative survival. Biometrics 2012, 68:113-120.

25. Galceran J, Puigdefabregas A, Ribas G, Izquierdo A, Pareja L, Marcos-Gragera $R$ : [Cancer survival trends in Catalonia and comparison with Europe]. Med Clin (Barc.) 2008, 131(Suppl 1):19-24.

26. Gispert R, Cleries R, Puigdefabregas A, Freitas A, Esteban L, Ribes J: [Cancer mortality trends in Catalonia, 1985-2004. Med Clin (Barc.) 2008, 131(Suppl 1):25-31. 
27. Esteban L, Cleries R, Langohr K, Galvez J, Pareja L, Escriba JM, Ribes J: [Statistical analysis of rates and trends (SART): a web-based tool for statistical calculation of population indicators]. Gac Sanit 2011, 25:427-431.

28. Valls J, Cleries R, Galvez J, Moreno V, Gispert R, Borras JM, Ribes JRD: A web tool for the analysis of the difference due to risk and demographic factors for incidence or mortality data. BMC Publ Health 2009, 9:473.

29. Cleries R, Valls J, Esteban L, Galvez J, Pareja L, Sanz X, Alliste L, Martinez JM, Moreno V, Bosch X, Borras JM, Ribes JM: WAERS: an application for Web-assisted estimation of relative survival. Med Inform Internet Med 2007, 32:169-175.

30. Davis ME, Phillips JA: Learning PHP and MYSQL. California, US: O'ReillyMedia Inc. Gravenstein; 2007.

31. R Development Core Team: $R$ : A language and environment for statistical computing. Vienna, Austria: R Foundation for Statistical Computing; c. http://www. R-project.org. 2008 Ref Type: Electronic Citation. ISBN ISBN 3-900051-07-0.

doi:10.1186/1471-2458-13-201

Cite this article as: Esteban et al: REGSTATTOOLS: freeware statistical tools for the analysis of disease population databases used in health and social studies. BMC Public Health 2013 13:201.

\section{Submit your next manuscript to BioMed Central and take full advantage of:}

- Convenient online submission

- Thorough peer review

- No space constraints or color figure charges

- Immediate publication on acceptance

- Inclusion in PubMed, CAS, Scopus and Google Scholar

- Research which is freely available for redistribution 